\title{
DIE NOMINALISIERUNG IN DER DEUTSCHEN UND INDONESISCHEN SPRACHE IM VERGLEICH
}

\author{
Eni Tiganni Purba \\ Siti Kudriyah \\ Jujur Siahaan
}

\begin{abstract}
AUSZUG
Das Ziel dieser Untersuchung ist es, die Nominalisierung aus den Adjektiven und aus den Verben in der magazin "Bild der Frau"Edition April/Mai Nr.2 2017 und Dezember Nr.49.2017. In dieser Untersuchung wird die deskriptive qualitative Methode verwendet. Die Datenquelle dieser Untersuchung ist die Nominalisierung aus den Verben und Nominalisierung aus den Adjektive verwendet. Die Untersuchung verwendete die Tecknik der Datensammlung, um die zu kennen, zu markieren, zu versammeln, zu analysieren, und zu Vergleichen mit den angesammelten die Nomen zu verallgemeinen. Die Ergebnisse dieser Untersuchung gibt es 222 die Nominalisierung, darunter sind 156 die Nominalisierung aus den Verben und 66 die Nominalisierung aus den Adjektiven. Das Ergebnis zeigt, dass die Nominalisierung aus den Verben mit dem Suffixen; ung, -er, -ion/tion, -nis, -n gebildet werden. Die Nominalisierung aus den Adjektiven mit dem Suffixen; -keit, -heit, -nis, -tät, -schaft gebildet werden.

Schlüsselwörter : Die Nominalisierung, Magazine"Bild der Frau"

\section{EINLEITUNG}

Die Morphologie ist der Teil der Linguistik, der die Feinheiten der Struktur untersucht. Es ist bereits an anderer Stelle darauf hingewiesen worden, dass ein Morphem die kleinste bedeutungs bestimmende Ausdruck sein heit ist, die wir kennen (Lado 1973:38).

Chaer (2015:3) behauptet, dass das Wort Morphologie aus den Worten morf was "Form" bedeutet und "logos", was Wissenschaft bedeuten. So bedeutet das Wort "Morphologie" die Lehre von der Form, also ist die Morphologi ein der Linguistik die Lehre von den Formen und der Bildung eines Wortes. Die Sprachwissenschft kennt mehrere Morphemtypen : Stämme oder Wurzeln, Flexions morpheme und Ableitungs morpheme (Lado,1973:39). Kridaklasana (2009:158) sagt, Morpheme sind die kleinste Einheit der Sprache. Sie können nicht in kleinere Abschnitte differenziert werden.

Die Identifizierung von deutschen Substantiven anhand ihrer morphologischen Struktur ist wie der Prozess der Wortbildung. Es wird 
untersucht, ob die Kompenenten ordnungs gemü $\beta$ sind und ob das Substantiv von anderen Wortklassen abgeleitet (Siahaan, 2013:4). Heringer (2014 : 198) erklärt, dass Nominalisierung Sachverhalte nicht in Satzform, sondern als Nominalphrase Formuliert. Das setzt voraus, dass ein Verb Nominalisiert wird als Infintiv und als Ableitung aus Verben oder Adjektiven.

Nominalisierungen sind eine Art von Wortbildungen. Im Deutschen kann die Wortbildung in Subtantiven, Verben, Adverbien und Adjektiven auftreten. Nominalisierungen sind sehr Wichtig, weiles die Nominalisierung in allen vier Fertigkeiten des Deutschen gibt. Die Studenten müssen den Bildungsprozess die Nominalisierung kennen. Ein Magazin ist für die Leser schwierig zu verstehen. Aberwenn die Bedeutung der Wörter oder Nominalisierung durch sucht wird, so dass den Lesern dann Schnell geholfen wird, um die Mittellung zu verstehen.

Der Bildungsprozess die Nominalisierung macht den Studenten des Deutschprogrammes manchmal Schwirigkeiten, weil es Verschiedene Bildungs prozesse die Nominalisierung zwischen Indonesich und Deutsch gibt. Je höher die Wissenschaft der Menschen ist, desto mehr neue Wortschatz muss beherrscht werden. Die Bildung des neuen Wortschatz wird in die Nominalisierung gelernt. Beim Deutsch lernen gibt es viele Nominalisierungen, zum Beispiel die Gesundheit, die Grosse, die Mahnung, und die Schreibe. Beim Indonesischen gibt es viele Nominalisierungen, zum Beispiel kesehatan, kebesaran, ketuhanan und kesederhanaan.

Das Magazine ist eine Publikation oder Veröffentlichung in regelmäßigen Abständen, die Artikel von verschiedenen Autorenenthält. (auf der Seitehttp://www.e-jurnal.com/2013/12/pengertian-majalah.html. gelesen am 20.4.2017 um 19.30 Uhr). Magazine enhält artikeln, die als Lesetexte zum Fremdsprachenlernen verwendet warden können. Mit Texten kann man sich über Wortbildungen beschäftigen. Basierend auf den obigen Beschreibungen ist es sinnvoll, die Nominalisierung in der deutschen und indonesischen Sprache zu analysieren und zu vergleichen.

\section{THEORETISCHE}




\section{UND KONZEPTUELLE GRUNDLAGE}

\section{Der Begriff des Wortes}

Die Bedeutung eines Wortes ist abhängig von der Satzsrtuktur, in der das Wort verwendet wird. Die Wortbedeutungen, die ein Wörterbuch angibt, können nur Anhalts punkte sein, weil die angegebenen Wörter aus bestimmten Satzstrukturen isoliert wurden. Nur bei übereinstimmten Satzstrukturen stimmen auch die Wortbedeutungen überein (Griesbach 1970:11).

Die Syntax ist die kleinste Einheit, in der morphologie, Sie ist die größte Einheit in der Morphologie, erstellt durch einen der morphologischen Prozesse (Affixation, Reduplikation, und Konvektion) (Chaer 2015:5).

Verhaar (1988:65) sagt, dass Derivation ein Affixprozess ist, durch den Indentitätwort verändert wird. Kridaklasana (1992:23) erläutert, das zusammengesetzte Wörter oder Komposita die Vereinigung von zwei oder mehr Leksemen ist, die ein Wort formen. Ein Nomen kann aus einen Nomen, Adjektiv oder Verb geformt werden. In diesem Prozess gibt die Elemente Suffix und Präfix, die eine grosse Rolle haben.

\section{Der Begriff der Subtantive}

Das Substantiv ist ein Wort, das ein Ding, einen Menschen, ein Tier, oder einen Begriff bezeichnet. Substantive werden im Deutschen mit einem grossen Buchstaben am Wortanfang geschrieben. Die meisten Substantive haben einen Singular - und eine Pluralform und können mit einem Artikel (der, die, das) verbunden werden, der auch das Genus anzeigt (Götz in Siahaan, 2013 : 21).Substantive bezeichnen personen, andere Lebewessen, konkrete Dinge sowie abstrakte Begriffe (Stang, 2014:11)

Beim Substantiv kann man nicht nur wie beim Artikel das Genus (Maskulin, Feminin, Neutrum), den Numerus (Singular, Plural) und den Kasus (Nominativ, Genitiv, Dativ, Akkusativ), sondern auch die Deklination bestimmen, denn die Substantive werden je nach Deklinationstyp unterschiedlich gebeugt, nämlich a. Genus, b. Numerus und c. Kasus. 


\section{Nominalisierung im Indonesischen}

Kridalaksana (2007 : 72) sagt, dass Nominalisierungen den Prozess der Bildung von Substantiven aus anderen Worten abgeleiteten Morpheme oder Klasse bezeichnet.Die Morphologie widmet sich also der Bestimmung und Klassifizierung von Morphemen und beschreibt die Kombinationstypen, die für die Wortbildung einer Sprache kennzeichnend sind (Lado, 1973:40). Es gibt die fünf Arten eine Morphologie zu andern : Affixation, Reduplikation, die internal veranderung, Supletion und die leere Modifikation (Samsuri, 1991:90).

Verhaar ( 1988 : 65) sagt, dass Derivation ein Affixprozess ist, um das Indentitätwort zu bilden, während Kridalaksana (2009:23) erläutert, dass Zusammengesetzte Wörter oder Komposition zwei oder mehr Leksemein einem wort vereinigen. Nomen können aus Nomen, Adjektiven und Verben gebildet werden.

Parera (1994:124-125) behauptet, dass die Morphologischen Prozesse sind Präfix, Suffix und Infix. Im Indonesischen Präfix sind pe-,ke-,men-,ber-,ter-, und di -. zum Beispiel pemuda, ketua, menguning, bersepeda,terpaku,und dipikir. Danach Suffix sind-an,-i,-kan,-nya,-si,-sang. Zum Beispiel manisan,kurangi,bukukan, luasnya, si manis, sang merah putih. Infix sindke-an und pe-an. Zum Beispiel ke-datang-an, per-kebun-an und ke-ibu-an.

\section{Nominalisierung im Deutschen}

Heringer (2014 : 198) erklärt dass, Nominalisierung Sachverhalte nicht in Satzform, sondern als Nominalphrase formuliert. Das setzt voraus, dass ein Verb nominalisiert wird : a. als Infintiv und b. als Ableitung aus Verben oder Adjektiven.

In der kompakten Nominalphrase wird das Prädikat des Nebensatzes zum nominalen Kern der Nominalphrase, die Objekte oder das Subjekt werden zu Attributen. Entsprechend kann man die Nominalisierung als Nebensatz auflösen: Attribute als Subjekt oder Objekt und das Verb als Prädikat (Heringer, 2014:198).

1. Unter Nominalisierung versteht man 
Die Bildung eines Nomens (Substantivs) aus einer anderen Wortart bzw. aus dem Wortstamm einer anderen Wortart. Zur Nominalisierung werden häufig

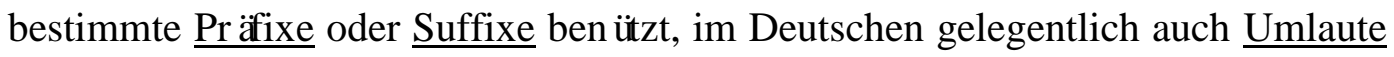
oder $\underline{\text { Ablaute. }}$ Im Deutschen kann man Verben und Adjektive/Adverbien nominalisieren.

2. Die Umformung verbaler Bestandteile eines Satzes in eine Nominalphrase.

Affixationist der Prozess der Bildung eines Wortes dargestellt vor allem für Substantive :

Eisenberg (2006: 663) sagt, Wortbildungsaffixe sind nicht wortfähige Wortbildungsmittel, die zusammen mit einer Derivationsbasis - das sind Stämme, syntaktische Fügungen oder Konfixe-komplexe Wortstämme bilden ( er-, ex-, ent-, -ig, -ität, -iv, -ier [en]). Sie sind nicht basisfähig, d.h. , sie können sich nicht mit ihresgleichen zu Stämmen verbinden (*er-lich, *unschaft). Nach ihrer Position im komplexen Stamm unterscheidet man Präfixe, Suffixe und Zirkumfixe.

Affixe sind Reihen bilden, d. h., prägen an Basiswörtern derselben Wortart eine Vielzahl von Wortbildungen mit derselben Wortbildungsbedutung aus wie beispielsweise -bar> kann [ Verb ] werden < an verbalen Basen : denk-, ess, hör-, verant-, wort-, vertretbar.

Bei der substantivischen Derivation sind alle drei Unterarten vertreten: die Suffixderivation (Maler), die Präfixderivation (Missernte) und Zirkumfixdervation (Eisenberg, 2006:731). Siahaan (2013:29) behauptet dass, die morphologischen Prozesse.

1. Präfix.

Verbale Präfixe können dagegen die Wortart verändern. Sie bildenPräfixderivate aus Verben, aber auch aus Substantiven und Adjektiven( Eisenberg, 2006: 665).

Ein für das Deutsche charakteristisces Wortbildungsmuster stellen die Präfixverben dar( z.B.besteigen,erwachen,auffessen,einziehen,ausstossen), die entweder als Ableitungen eingeordnet oder(wegen der Komplexitat der 
Gruppe) als eigener dritter Typus neben Zusammensetzung und Ableitung angesehen werden.

Die Präfixe haben die Funktion, den Verbinhalt zu modifiezieren. Sie drüken entweder die Richtung aus (einziehen,ausstessen) oder die Aktionsart, das bedeutet vor allem die zeitliche Verlaufsweise. Eines Seins oder Geschehens: Verben in denen die zeitliche Begrenzung eines Geschehens ausgedruckt ist, nennt man perfektiv (Bünting, 1990:122-123). Das NomenPräfix ist un;miss,ertz;Un

2. Suffix

Suffixe treten rechts an die Basis und Bilden mit ihr einen komplexen Wortstamm. Die meisten nativen Suffixe sind unbetont ( außer-ei/-eirei), bei Fremdsuffixen gibt es sowohl betonte (-iv in alterna'tiv, -ation in Genera'tion) als auch unbetonte (-ik in 'Lyrik) und auch enterprechende Derivate mit wechselnder Betonung (-or in 'Lektor, Lek'toren). Eisenberg(2006: 665). Sufix ist ein gebundenes Morfem, und liegt am Ende des Grundswortes(Siahaan, $2013: 33$ )

Nomensuffixe in der deutsche Sprache bestehen aus: -keit/-heit,-e,-ität,ung,-(er)ei,-ist,-nis,-er,-at,-ei,-in,-or und -chen.

Um Nomen aus Verben abzuleiten gibt es die Suffixe -ung, -er, -ling, -nis, - erei, -e. Nomen werden aus Adjektive abgleitet mit Suffixen -chen, -lein, -le, schaft, -tum, - heit, -er, -in,-ner,- ler,-ler,-ist

Aus den Begriffen kann man zusammengefasst, dass ein Verb zu einem Nomen Umgewandelt, Spricht man von einer Nominalisierung. Umgekehrt Spricht man von einer Nominalisierung,wenn das Nomen zu einem Verb umgewandelt werden.

\section{Das Magazine}

Das Magazin ist eine Medien-Veröffentlichung oder periodische Publikation von Texten von verschiedenen Autoren, (Assegaff, 1983). Ein Magazin enthält Artikel, Kurzgeschichten, Bilder, Bewertungen, Illustrationen, die den Inhalt des Magazins charakterisieren. (auf der Seite http://www.ejurnal.com/2013/12/pengertian-majalah.html gelesen 20.4.2017 um 18:33 Uhr) 
F. Frazier Bond behauptet, dass es zwei Arten von Magazinen gibt:Öffentliche Magazine und Spezielle Magazine.

Ein Magazin ist Print medien oder Elektonische Publikationen(Elektronische Zeit Schrift) die in( regelmässigen oder Unregelmässigen) Abständer in gleicher, gehefter oder gebundener Form erscheinen.Oft wird auch der Begriff "Magazin" verwendet.

Im Gegensatz zu Zeitungen sind die weniger auf Nachrichten, sondern mehr auf Hintergrund berichterstattung zu aktuellen Themen ausgelegt. Meist Konzentriert sich eine Zeitschrift auf einem bestimmten Themen bereich, dagegen ist die geograpische Lage und Verbreitung Irrelevant, anders als bei den moistens Tages Zeitungen.(www.Industriedruck.de/hefte.html gelesen am 22april 2017).

Auf der Seite www.Magazine.deutschland.de, kann man herausfinden dass "Magazin Deutscland" eine deutschsprachige Zeitschrift ist, die in zusammen arbeit mit dem Auswärtigen Amt und der Frankfurter Societäts Medien Gmbh in 180 Ländern vertrieben wird. Unter der Leitung des cheftredakteurs Peter Hintereder arbeitet ein Team von bekannten nationalen und Internationalen Journalisten und auch Wissenschaft lern Zusammen.

\section{UNTERSUCHUNGSMETHODOLOGI}

In dieser Untersuchung wird die Qualitätiv methode verwendet. Die Untersuchung wird durch geführt, um die Nominalisierung im Magazin "Bild der Frau" zu analysieren.

\section{Die Daten und Datenquelle}

Die Daten in dieser Untersuchung sind Adjektiven, und Verben zum Thema Gesundheit im Magazin“Bild der Frau” (Nr. 2 April/Mei 2017 und Nr. 49 Dezember 2017). Die Datenquelle der Untersuchung ist das Magazin "Bild der Frau" (Edition Nr. 2 April/Mei 2017). Dieses Magazin hatin gesamt 173 Seiten.

\section{Die Technik der Datenanalyse}


Für die Datenanalyse in dieser Untersuchung wird das Qualitativ Verfahren Verwendet. Die Schritte der Datenanalyse warden nach der definition von Miles und Hubermann (2014:178).

\section{ERGEBNISSE DER UNTERSUCHUNG}

\section{Die Nominalisierung}

1. Die Nominalisierung aus den Verben

Nach der Datenanalyse werden 156 Nominalisierungen aus denVerben identifiziert. Daraus werden 102 mit Sufifx -ung 28 Nominalisierungen mit Suffix -er, 18 Nominalisierungen mit Suffix -ion/tion und 6 Nominalisierungen mit Suffix - $\boldsymbol{n}$ is, und 2 Nominalisierungen mit Suffix -ngebildet.Die folgenden sind Beispielen von Nominalisierungen, die von einem Suffix abgeleitet sind:

1. Die Sendung stammt aus einem Verb "senden" mit ein Suffix - ung.Das Verb“senden"bedeutetpengiriman. Das Wort pengiriman stammt aus “ kirim” mit Konfix pe + (NG) ...+ an gebildet. Vergleichbare Nominalisierung im Indonesischen sind pengurangan. Das Wort pengurangan wird von " kurang" mit Konfix pe + (NG)...+ an gebildet. Andere gleiche Beispiel dafür sind pengombinasian und pengembalian.

2. Die Besucherstammt aus einem Verbbesuchen mit Suffix-ergebildet. Das Verbbesuchenbedeutet “pengunjung”. Das Wort pengunjung wird von “ kunjung “ mit Präfix pe.Die Buchstabe “ k” (kunjung) wechslt auf “ ng” (ngunjung).Vergleichbare Nominalisierung im Indonesischen ist pengemudi. Das Wort pengemudi wird von “ kemudi” mit Präfix pe gebildet. Andere gleiche Beispiele dafür sind pengarang und pengirim.

3. Die Kombination stammt aus einem Verbkombinieren mit suffix-ion. Das Verbkombinierenbedeutet “ kombinasi”. Vergleichbare Nominalisierung im Indonesischen ist klasifikasi ( die Klasifikation). Das Wort klasifikasi wird den Verben “ klasifizieren" mit Suffix -ion gemacht. Andere Beispiel ist modifikasi (die Modifikation).

4. Das Verhältnis stammt aus einem Verbverhaltenmit Suffix-nis.Das Verb “verhalten"bedeutetkelakuan.Das Wort "kelakuan" wird von “laku”, mit Konfixke+...+an gemacht. Vergleichbare Nominalisierung 
im indonesischen ist kelengkapan .Das Wort kelengkapan wird von “ lengkap”, mit Konfix ke+...+an gebildet. Andere Beispiel dafür sind kelangsungan und kelancaran.

5. Das Vermittelnist eine Nominalform und stammt aus einem Verb “vermitteln", das penghubungan bedeutet. Zum Nominalisieren benutzt man das Verb “vermitteln” . Vergleichbare Nominalisierung im indonesischen ist penghilangan.

2. Die Nominalisierung aus den Adjektiven.

Nach der Datenanalyse werden die 66 Nominalisierungen aus den Adjektiven identifiziert. Daraus werden 40 mit Suffix -keit gebildet, 15 Nominalisierungen aus den Adjektiven mit Suffix -heit und2 Nominalisierungen mit Suffix -nis, und6Nominalisierungen mit Suffix - tät , 3 Nominalisierungen mit Suffix -schaft.Die folgenden sind Beispielen von Nominalisierungen, die von einem Suffix abgeleitet sind:

1. Die Traurigkeitstammt aus einem Adjektive "traurig" mit Suffix-keit.Das Adjektivtraurig bedeutet"kesedihan”.Vergleichbare Nominalisierung im Indonesischenistkesetiaan. Kesetiaan stammt aus ein Adjektiv "setia"und wirdmit Konfix ke $+\ldots+$ angebildet. Andere gleiche Beispiele dafür sind kesederhanan und kesempatan.

2. Die Schönheit eine Nominalform und stammt aus einem Adjektiv "schön”, das kecantikanbedeutet. Das Wort kecantikan wird von “cantik” mit Konfix ke +...+an gebildet. Vergleichbare Nominalisierung im Indonesischen kecemburuan, das aus Adjektiven “cemburu” mit Konfix ke+...+angebildet wird. Andere Beispiele dafür sind kepentingan und kepedulian.

3. Die Bereitschaft stammt aus einem Adjektive bereitmit Suffix-schaft. Das Adjektiv " bereit" bedeutet"siap". Das Wort kesiapan wird von "siap" mit Konfix ke +...+an gebildet wird. Vergleichbare Nominalisierung im Indonesischenistkesanggupan, das aus Adjektiven “ sanggup”mit Konfix ke 
-angebildet wird. Andere Beispiele dafür sind kesesuaian, kesendirian und kesiangan.

4. Die Elastizität stammt aus einem Adjektiv elastiz mit Suffixtät. Das Adjektivelastiz bedeutet kualitas.Zwischen dem Wortstamm “elastiz” und dem Suffix tät wird eine Silbe “i” hinzugefügt. Vergleichbare Nominalisierung im indonesischen istregional (die Regionalität).

5. Das Gedächtnis eine Nominalform und stammt aus einem Adjektiv "gedacht"das bedeutet kenangan. Das Wort kenangan wird von “kenang” mit Suffix $(\ldots+$ +an) gebildet. Vergleichbare Nominalisierung im Indonesischenistkeluhan.Keluhan stammt aus Adjektiven "keluh"mit Suffix (...+an)gebildet. Andere Beispiel sind kerutan und kebutan.

\section{Diskussion}

Besierend auf Ergebnisse die Nominalisierung in der Deutschen und Indonesischen sprache im Vergleichen wurde zusammengefasst, dass die Daten in dieser Untersuchung aus dem Magazine, Internet, Buch, und einigen Website genommen warden. In dieser Unterschung wird Suhardi in Indonesischen Teori benutzt und in Deutsch Hans Jürgen Heringer Theori benutzt. Dieser Untersuchung mit Analyse die Nominalisierung im Magazine ist gut, denn die Werte Molarosierung gibt, und ist und ist interessant. So das Dosentinen oder die Studenten das als Lernmaterial benutzen kann.

\section{SCHLUSSFOLGERUNG}

Nach den Untersuchungsergebnissen fallen die Schlussfolgerungen folgendermassen aus: Es gibt 222 die Nominalisierung in der Magazine " Bild der Frau “ Edition Nr.2 April/Mai 2017 und Nr.49 Dezember 2017; darunter sind 156 Nominalisierungen aus den Verben und 66 Nominalisierungen aus den Adjektiven.

1.) Es gibt 156 Nominalisierungen in der deutschen aus den Verben, zum Beispiel: die Wirkung, die Richtung, die Überforderung, die Ernährung, die Bildung, die Lösung, die Haltung, die Wohnung, die Verwertung und die Übung. Ähnliche Nominalisierung im indonesischen sind unter anderen: penulisan, 
penerimaan, penganiayaan, penginspirasian, pemberian, pemberangkatan, pembimbing, pembeli,berduaan, und bacaan.

2.)Es gibt 66 Nominalisierung in der deutschen aus den Adjektiven, zum Beispiel: die Feuchtigkeit, die Beweglichkeit, die Geschwindigkeit, die Wendigkeit, die Schnelligkeit, die Neueheit, die Sicherheit, die Gesundheit, die Traurigkeit, und die Profesioneller. Ähnliche Nominalisierung im indonesischensind unter anderen: kekeringan, kekuatan, ketakutan, peganugrahan, kedisplinan, kelamaan, ketentuan, kesedihan, kebaikan und kepintaran.

3.) Der Vergleich von Nominalisierung der deutschen und indonesischen kann folgendes beschrieben werden; die Nominalisierung im deutschenbesteht.

aus Nomen, die aus dem Suffix und dem Wortstamm gebildet werden. Während im indonesischen aus Nomen besteht, aber das Suffix und Wortstamm nicht hinzufügefügt werden. Nominalisierung der indonesischen besteht aus dem Wortstamm. Die Nominalisierung der deutschen können zwei Wörter zusammen gesetzt werden, das sind: die Fettverbrennung und die Bewegungsrichtung, während indonesische von einem Nomen Wort gebildet wird. Nominalisierung der deutschen kann zwei von einem Suffix (lich, keit) gebildet werden, das sind: die Beweglichkeit, die Verlässlichkeit, die Feuchtigkeit, und die Nierentätigkeit, während die indonesischen Nomen von einem Suffix gebildet wird. Es gibt Nomen, die von 3 Suffixen das ist: los, ig, keit zusammengesetz werden, zum Beispiel: die Langzeitarbeitlosigkeit.

\section{LITERATURVERZEICHNIS}

Assegaff,Djafar.H.2006. Jurnalistik Masa Kini (Pengantar Praktek

Kewartawanan).Jakarta: Ghali Indonesia.

Bünting,K.-D .1990. Einführung in die Linguistik.Fankfurt.( im materilien von

Grundkurs der Sprachwissenschaft).

Chaer, Abdul.2015. Morfologi Bahasa Indonesia. Jakarta:Rineka Cipta.

Eisenberg, Peter.Et al. 2006. Duden die Grammatik 4. Wien:Duden Verlag. 
Studia: Journal des Deutschsprogramms p-ISSN 2301-6108 e-ISSN 2654-9573

https://jurnal.unimed.ac.id/2012/index.php/studia/index

Griesbach, Heinz. 1970. Deutche Grammatik im Überblick. München: Max Hueber Verlag.

Heringer, Hans Jürgen. 2014. Deutsche Grammatik und Wortbildung in 125 Fragen und Antworten.Tübingen: A.Francke Verlag.

Kridalaksana, Harimurti.2007. Kelas Kata Dalam Bahasa Indonesia Edisi Kedua. Jakarta:PT Gramedia Pustaka Utama.

Kridalaksana, Harimurti. 2009. Pembentukan Kata dalam Bahasa Indonesia. Jakarta:PT Gramedia Pustaka Utama.

Lado, Robert .1973. Moderner Sprachunterricht. Eine Einfuhrung auf Wissensechaftlicher Grundlage. München, Germany : Max Hueber Verlag.

Magazine Bild der Frau Schlank \& Fit Nr.2 April - Mai 2017.

Miles und Huberman. 2014. Metode Penelitian Deskriptif. Jakarta: P.T. Gramedia Pustaka Utama.

Parera,Jos Daniel .1994 . Morfologi Bahasa. Jakarta: P.T . Gramedia Pustaka Utama.

Siahaan, Jujur. 2013. Analisis Morfologis Nomina Bahasa Jerman. Medan:Unimed Press.Samsuri. 2003. Analisis Bahasa. Jakarta: Erlangga.

Stang, Christian.2014.Die Neue Rechtschreibung Kurz und einfach. Köln: Anaconda

VerlagGmbH.

Verhaar, J.W.M. 1982. Pengantar Linguistik.Yogyakarta: Gadjah Mada University

Press.

http://19.uhamzah.web.id/id3/2823-2721/Nominalisasi_24783_19-uhamzah.html (gelesen am 20.4.2017,um 19.20 Uhr). 
Studia: Journal des Deutschsprogramms p-ISSN 2301-6108 e-ISSN 2654-9573 https://jurnal.unimed.ac.id/2012/index.php/studia/index

http://www.e-jurnal.com/2013/12/pengertian-majalah.html (gelesen am 20.4. 2017,um $18: 33$ Uhr).

http://www.deutschegrammatik20.de/deutsche-grammatikinhaltsverzeichnis/glossar-grammatik/glossar-n/grammatikglossarnominalisierung/ (gelesen am 10.7.2017, um $15: 30$ Uhr).

http://suz.digitaleschulebayern.de/deutsch/dunterlagen/substantiv theorie.pdf(gele sen am 10.7.2017, um 16 :05 Uhr).

http://mein-deutschbuch.de/nominalisierung.html (gelesen am 22.4.2017, um 15 :00 Uhr). 